\title{
An Automated 3D Algorithm for Neo-cortical Thickness Measurement
}

\author{
S. Srivastava, F. Maes ${ }^{\star}$, D. Vandermeulen, P. Dupont, W. Van Paesschen, and \\ P. Suetens
}

\begin{abstract}
Katholieke Universiteit Leuven, Faculties of Medicine and Engineering, Medical Image Computing (Radiology - ESAT/PSI), University Hospital Gasthuisberg, Herestraat 49, B-3000 Leuven, Belgium.

Siddharth.Srivastava@uz.kuleuven.ac.be
\end{abstract}

\begin{abstract}
A new method is presented for fully automated regional quantification of human neo-cortical thickness from 3-D MR images. The method does not require explicit extraction of the cortical surface, but instead is based on a geodesic distance transform of the binarized 3D volumetric gray matter map obtained by automated segmentation. Regional cortical thickness distributions are derived by atlas-based partitioning of the brain. Results for 64 normal controls are presented that show very good agreement with published reference values.
\end{abstract}

\section{Introduction}

A pathological change in the thickness of the human cerebral cortex is associated with a wide variety of neurological and pathological disorders. The motivation for this work is focal cortical dysplasia (FCD), which is a malformation of cortical development (MCD) characterized by dyslamination, abnormal cortical components, blurring of the gray/white matter interface, and an increase in cortical thickness at the site of the lesions 10. A typical case is illustrated in figure 1] Because subtle FCD lesions may not be noticed with standard radiological examination, we are working towards a method that is able to accurately detect and localize FCD lesions automatically from MR images, based on the detection of regions with abnormal thickness values.

In this paper, we focus on reliable, automated measurement of cortical thickness in normal brains within various regions of interest. Most of the approaches for cortical thickness measurement from 3-D images that have been proposed in the literature require an explicit reconstruction of the geometry of the cortical surface [6] and rely on accurate segmentation of the inner and outer cortical surfaces, which is error prone. In contrast, the method we propose in this paper does not require explicit cortical surface segmentation, but instead is based on a geodesic distance transform acting directly on gray matter (GM) segmentation map represented as a 3-D voxel volume, as described in Section 2 Section

\footnotetext{
* Frederik Maes is Postdoctoral Fellow of the fund for Scientific Research Flanders(FWO-Vlaanderen, Belgium).
} 


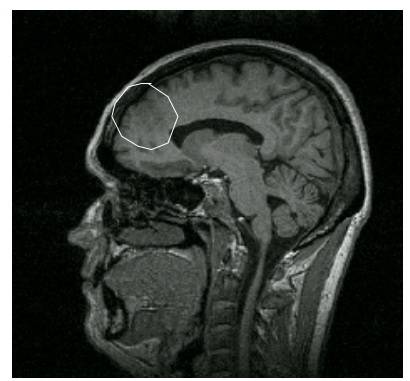

(a)

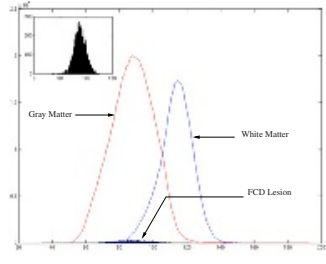

(b)

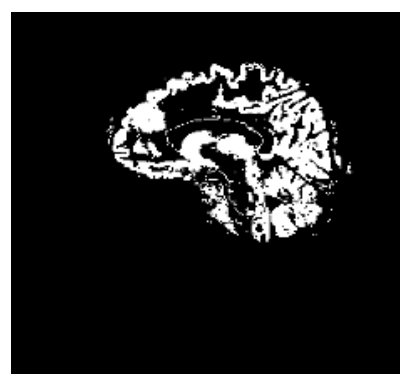

(c)

Fig. 1. (a)Patient MR image with a subtle FCD lesion marked; (b): Intensity histogram of the lesion in relation to those of gray and white matter. The inset shows a magnified view of the lesion intensity histogram. (c) :Binarized segmented gray matter tissue map of (a), which shows that the lesion has been classified as gray matter.

3 describes how regional cortical thickness distributions are extracted by automated and consistent atlas-based partitioning of the brain using non-rigid atlasto-patient image matching. Regional thickness values from 64 normal controls are presented in Section 4 and compared with published reference values.

\section{Cortical Thickness Measurement}

Our approach for cortical thickness measurement in the scope of FCD lesion detection is based on two assumptions. Firstly, we assume that the intensity of range of the FCD lesions in T1-weighted MR images largely overlaps with that of gray matter itself, such that the FCD lesions are labeled as gray matter by an intensity-based tissue classification algorithm. Figure 1 illustrates a typical case that justifies this assumption. Consequently, with the lesions completely included in the segmentation of the cortex, FCD lesions can be detected as areas within the segmented cortex with abnormal thickness. Secondly, we assume that the segmented outer cortical layer or neo-cortex (which excludes the deep gray matter structures) is locally topologically equivalent to the spherical shell, whose thickness is small compared to its inner radius. The thickness of the cortex can therefore be measure by propagating distances from the outer cortical surface (the GM/CSF interface) within the domain of interest (GM) towards and onto the inner surface (GM/WM interface), or vice-versa. Our approach for FCD lesions detection thus consists of two steps: (1) segmentation of the cortex from T1-weighted MR images by intensity-based pixel classification; (2) thickness measurement of the cortex by a distance transform of the binary cortical region obtained by segmentation.

The cortex is segmented using the fully automated model-based MR brain image segmentation algorithm proposed by Van Leemput et. al. [5]. This segmentation algorithm assigns each voxel a probability to belong to a particular brain tissue type (WM, GM, CSF or other) based on its intensity and spatial 
context, after bias field correction to account for possible MR intensity inhomogeneity. The intensity histogram of each tissue class is modeled as a Gaussian distribution with unknown mean and spread, which are estimated from the data simultaneously with the classification and the bias field. Starting from an initial classification provided by a digital atlas that is matched to the patient images by image registration [7], the algorithm uses an Expectation- Maximization approach to maximize the likelihood of the image data given the model parameters, iteratively alternating between estimating the model parameters and recomputing the classification using updated parameters. The resulting probabilistic tissue maps are subsequently binarized by assigning each voxel to its most likely tissue type. A typical segmentation result is illustrated in figure 2 .

The Euclidean distance transform of the binary object $\mathcal{X}$ assigns to each point $x$ of $\mathcal{X}$ its distance $\mathcal{D}_{\mathcal{X}}(x)=\min _{y \in X^{c}}\|x-y\|$ to the background $\mathcal{X}^{c}$. When applied to segmented GM object $\mathcal{G}$ directly, a medial axis transform of the cortex is obtained, which is not desired, as it fails to assign the largest values to the FCD lesion itself and therefore does not allow to discriminate the lesion. BY constructing the union of the binarized GM and WM objects $\mathcal{G}$ and $\mathcal{W}$, a distance map $\mathcal{D}_{\mathcal{G} \cup \mathcal{W}}$ relative to the GM/CSF interface is obtained, from which cortical thickness values $T_{\mathcal{G}, 1}=\mathcal{D}_{\mathcal{G} \cup \mathcal{W}} \times \mathcal{G}$ can be extracted by masking with the GM object itself. Similarly, distance values relative to the GM/WM interface can be obtained within the cortical domain by considering the white matter object $\mathcal{W}$ as the background and its complement $\mathcal{W}^{c}$ as the object of interest: $T_{\mathcal{G}, 2}=\mathcal{D}_{\mathcal{W}^{c}} \times \mathcal{G}$. However, considering $\mathcal{G} \cup \mathcal{W}$ or $\mathcal{W}^{c}$ obliterates the cortical features at the GM/WM or GM/CSF interface respectively. The propagation of distance values when calculating $T_{\mathcal{G}, 1}$ of $T_{\mathcal{G}, 2}$ is not restricted to the cortical domain $\mathcal{G}$ only, such that the thickness values obtained at the inner or the outer cortical surface respectively are likely to be underestimated. Hence a geodesic distance transform (see [1] and references therein) within the object $\mathcal{G}$ is required, that computes distances from the inner to the outer cortical surfaces or vice-versa along paths that are entirely contained in $\mathcal{G}$. The algorithm that we used to calculate the geodesic distance transform was presented by Cuisenaire 1], which is fast and accurate and can handle regions of sharp bends and corners, which are features that appear frequently in the cortical object. The algorithm is based on morphological dilation of the inner towards the outer surface (referred to as Mode 2 propagation) or vice-versa (Mode 1 propagation) using a ball shaped structuring element $B_{d}$ of radius $d$. Locally, within the neighborhood $B_{d}$, Euclidean distances are computed along straight lines, while structures at scales larger that $d$ may reorient the direction of distance propagation. The pseudo-code for the algorithm can be found in 11, which we implemented in $\mathrm{C}$ on a Linux workstation. Results for both modes of propagation are shown in figure 2

To quantify geodesic distance error propagation with successive dilations, a 2-D software phantom was created with surface undulations similar to that of the cortical layer and with sufficient thickness variation along its surface. The phantom is specified mathematically by $(x, y)=c+(r+\gamma \sin (\alpha \theta)) \cdot(\cos \theta, \sin \theta)$, which represents family of curves parameterized by $\theta$ and centered at $c$. $\alpha$ and $\gamma$ control the frequency and height of the undulations of the curve around the 


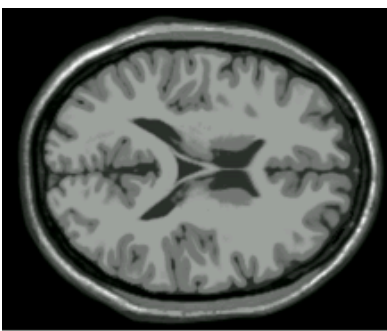

(a)

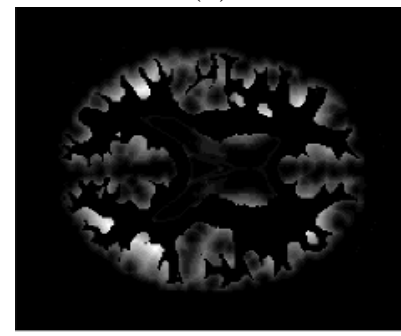

(d)

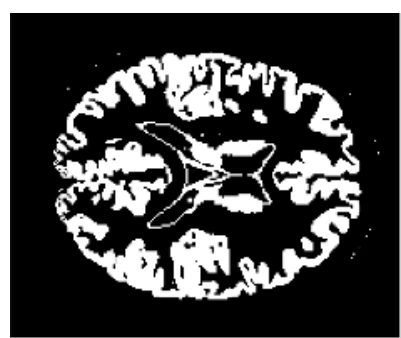

(b)

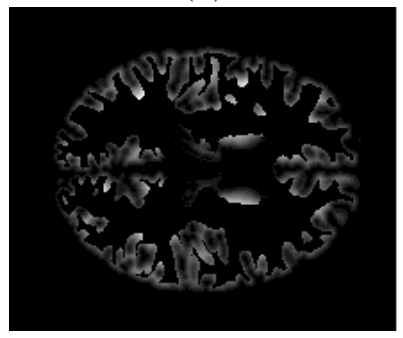

(e)

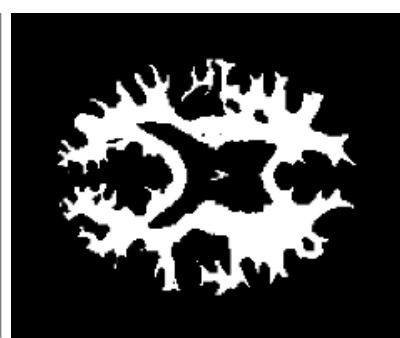

(c)

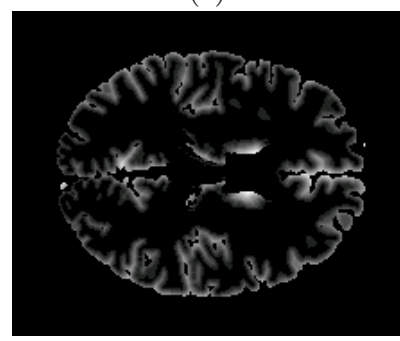

(f)

Fig. 2. (a) Axial slice of a 3-D weighted MR image, (b,c) Binary GM and WM obtained by tissue classification; (d) Euclidean unconstrained distance transform $T_{\mathcal{G}, 1}$ of the union of (b) and (c), masked by (b); (e,f) Geodesic distance transform of (b) obtained with outside to inside and inside to outside propagation respectively.

circle with radius $r$. Figure 3(a) shows 2 members of this family, labeled $R_{1}$ and $R_{2}$, with identical $c, \alpha$ and $\gamma$, but different $r$. The curves are first binarized on a discrete pixel grid, by setting all pixels hit by the curve to 1 . The $B_{d}$ geodesic distance transform with $d=1$ of the annular region $\mathcal{A}$ in between both curves is then computed by successive dilations of the inner curve $R_{1}$ outwards into $\mathcal{A}$ and onto the outer curve $R_{2}$. Because $R_{1}$ and $R_{2}$ differ by a scaling factor only, the propagation of distances proceeds along straight lines trajectories. Hence, for each pixel in $\mathcal{A}$, we compare the distance value generated by the geodesic distance transform with its true euclidean distance to the binarized curve $R_{1}$, calculated by an exhaustive search over all pixels of $R_{1}$. As shown in figures $3(\mathrm{~b}, \mathrm{c})$, the average error increases as the size $\left|R_{1}-R_{2}\right|$ of the phantom increases and the distances need to be propagated further, but the average error is smaller than 0.3 pixels for all the cases considered. For $\left|R_{1}-R_{2}\right|$ up to 6 pixels, which is the expected thickness range of the cortex in $1 \mathrm{~mm}$ isotropic MR images, almost all pixels have errors less than 0.2 pixels. Similar results were obtained when reversing the propagation direction, using successive dilations of the outer curve $R_{2}$ inwards into $\mathcal{A}$ and onto the inner curve $R_{1}$. A detailed error analysis of the distance transform algorithm can be found in [1]. 


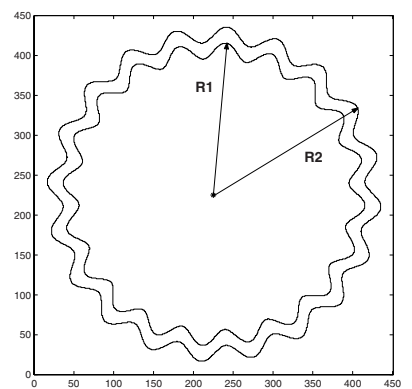

(a)

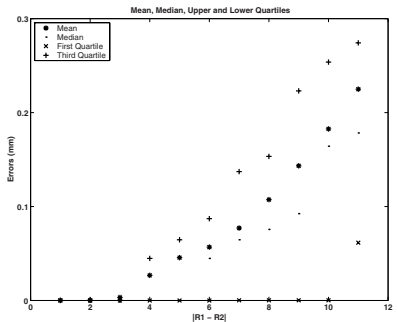

(b)

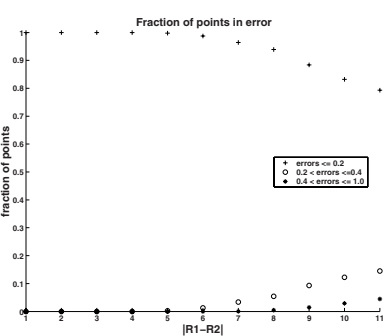

(c)

Fig. 3. (a) 2-D phantom mimicking the undulations of the cortex used for validation of the distance transformation; (b) Mean value and 25, 50 and $75 \%$ percentiles for the error between the estimated and the true distances to the inner curve $R_{1}$ in the annular region between $R_{1}$ and $R_{2}$, for various phantom sizes $\left|R_{1}-R 2\right|$. (c) Fraction of pixels with errors in ranges $[0,0.2],[0.2,0.4]$ and $[0.4,1.0]$ pixels for various phantom sizes.

\section{Regional Cortical Thickness of Normal Controls}

We applied our algorithm to measure cortical thickness in 64 normal controls from $1 \mathrm{~mm}$ isotropic high resolution T1-weighted images. In all cases, Mode 2 propagation was used. A regional analysis of cortical thickness was performed after partitioning the distance map in various cortical regions. Partitioning is performed automatically by atlas-based segmentation of each subject's MR image by non-rigid registration with a pre-segmented template brain image provided by Hammers [3], in which 49 volumes of interest (VOI) are individually labelled. The template is spatially normalized in Talairach space and is aligned with SPM99 [8] MR brain template. Each subject MR is first affinely registered to atlas by maximization of mutual information [7] and subsequently non-rigidly matched to atlas using non-linear spatial normalization routines of SPM99 8]. The resulting deformation is inverted using the Deformations Tolbox provided with SPM99 [8], and the inverse transformation is applied to the VOI template to warp it to the space of the subject's MR. Nearest neighbor interpolation is used to reformat the VOI template to the domain of the subject MR to ensure that each voxel is assigned to a unique VOI label. With Mode 2 propagation, the geodesic distance map needs to be sampled at the outer cortical layer within each region to obtain regional thickness distributions. The outer cortical layer is extracted as the set of all voxels that are assigned a distance between 0 and 1 voxels in Mode 1 propagation. These voxels are then masked by the VOI template in the space of the subject MR to obtain per-region thickness distributions.

\section{Results}

The cortical thickness map for a single subject is illustrated in figure 4 (a), while the cortical thickness distribution for the entire brain obtained by pooling all measurements over all 64 controls is shown in figure 4(b). The mean cortical 


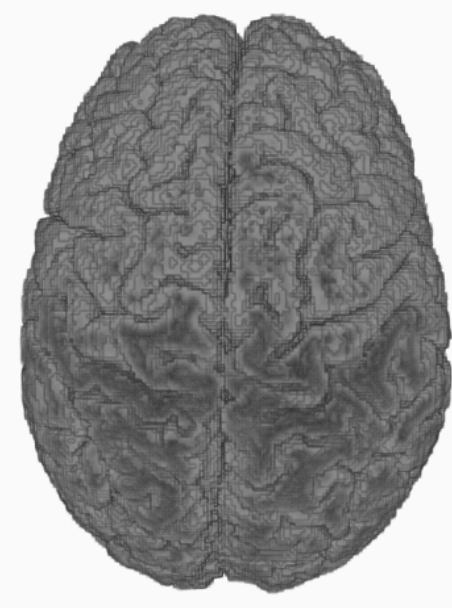

(a)

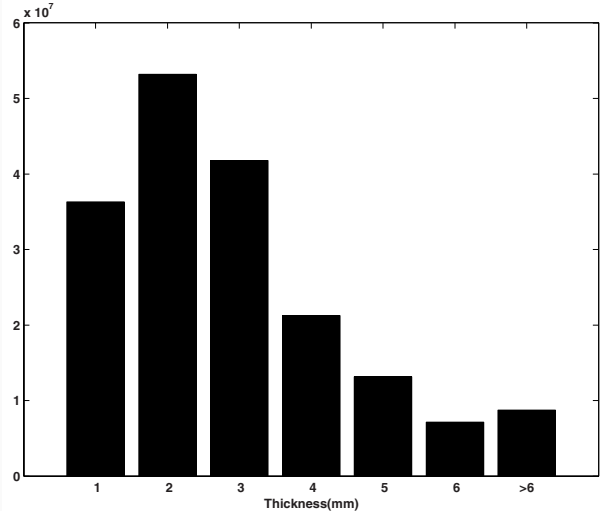

(b)

(c)

Fig. 4. (a) Cortical thickness map for a single subject with thickness values projected on the outer cortical surface using Mode 2 propagation, and color coded according to color map (c). (b) Thickness distribution for the whole brain, accumulated over all 64 controls. Values larger than $6 \mathrm{~mm}$ were accumulated and labeled as such.

thickness was $2.74 \mathrm{~mm}$, with a standard deviation of $1.8 \mathrm{~mm}$ and a median value of $2.5 \mathrm{~mm}$. These values agree very well with the measurements of von Economo [11, who reports cortical thickness to be between 1.2 and $4.5 \mathrm{~mm}$ with a mean value of $2.5 \mathrm{~mm}$. $95.7 \%$ of the cortical thickness distribution of figure 4 is contained within $6 \mathrm{~mm}$, and $92.2 \%$ of the distribution is contained within $5 \mathrm{~mm}$, which is also in close agreement with the values reported in [2. Mean and Median regional thickness values over all subjects for some of the 49 VOI included in the atlas are tabulated in Table 1

Table 2 compares our results with the measurements reported in the reference work on cyto-architectonics of the cerebral cortex by von Economo 11, and with the results of Kabani et. al. 4, who validated the automated method of [6] by comparisons with manual measurements. We have presented results only for those regions for which Hammers [3] parcellation overlaps with those of von Economo 11 and Kabani et. al. 4. A column labeled "corrected" has also been added to correct for $25 \%$ volume shrinkage (10\% thickness shrinkage) for post-mortem studies 9], by adding $10 \%$ to the values reported by von Economo. Our results agree with those of von Economo for all regions, except for Occipital Lobes. The thickness values for the insular region, reported as a difficult structure to measure [4] is also closer to von Economo's values than those of Kabani et. al. 
Table 1. Mean and median of the thickness (in $\mathrm{mm}$ ) some of the cortical regions of interest.

\begin{tabular}{|c|c|c|}
\hline Region & Mean (mm) & Median $(\mathrm{mm})$ \\
\hline R Frontal Lobe & 2.80 & 2.50 \\
\hline L Frontal Lobe & 2.97 & 2.65 \\
\hline R Parietal Lobe & 2.58 & 2.33 \\
\hline L Parietal Lobe & 2.67 & 2.50 \\
\hline R Temporal Gyri MiddleInferior & 3.22 & 3.15 \\
\hline L Temporal Gyri MiddleInferior & 3.19 & 3.15 \\
\hline R Lateral Anterior Temporal Lobe & 3.84 & 3.68 \\
\hline L Lateral Anterior Temporal Lobe & 3.82 & 3.68 \\
\hline R Superior Temporal Gyrus & 3.47 & 3.36 \\
\hline L Superior Temporal Gyrus & 3.28 & 3.15 \\
\hline R Occipital Lobe & 3.24 & 2.91 \\
\hline L Occipital Lobe & 3.43 & 3.15 \\
\hline R Lateral Occipitotemporal Gyrus & 3.21 & 3.15 \\
\hline L Lateral Occipitotemporal Gyrus & 3.23 & 3.15 \\
\hline R Insula & 3.70 & 3.36 \\
\hline L Insula & 3.67 & 3.36 \\
\hline R Cerebellum & 3.72 & 3.36 \\
\hline L Cerebellum & 3.82 & 3.47 \\
\hline
\end{tabular}

Table 2. Comparison of thickness values obtained by our method with those of von Economo 11 and Kabani et. al. 44. The column labeled "corrected" are von Economo's measurement corrected for $10 \%$ shrinkage

\begin{tabular}{|c|c|c|c|c|}
\hline Region & von Economo & Corrected & Our Method & Kabani \\
\hline Insula & $2.8-3.5$ & $3.0-3.9$ & 3.36 & 4.9 \\
\hline Post Cingulate & $2.5-3.0$ & $2.75-3.3$ & 2.95 & 3.9 \\
\hline Anterior Temporal Lobe & $3-4$ & $3.3-4.4$ & 3.68 & - \\
\hline Occipital Lobe & $1.2-2.5$ & $1.3-2.75$ & 3.03 & - \\
\hline
\end{tabular}

\section{Discussion}

We present an efficient and completely automated method for the accurate quantification of the human cerebral cortical thickness from 3-D MR images, based on a geodesic distance transform of the binarized gray and white matter object extracted by intensity-based brain tissue segmentation. Regional analysis of cortical thickness is performed by atlas-based partitioning of the brain after non-rigid registration with a VOI template.

The main source of error in our approach stems from segmentation errors. Misclassified partial volume pixels result in violation of topological assumption, leading to a local underestimation of thickness. For regions that are normally thin, like the occipital lobes, partial volume may cause overestimation of thickness values for these regions. Further, inaccuracies in the VOI templateto-subject warping affects the accuracy with which the VOI template covers 
the subject brain. In addition to these, regions which show large variation in thickness values along their extent can not be well represented by a single value.

We presented results for regional cortical distribution in 64 normal subjects. A fundamental problem is the lack of a proper ground truth for validation. The most widely used and well documented methods are on post-mortem brains, using Cavelieri sections, which, however, can not exploit 3D connectivity. Nevertheless, our results are in close agreement with those reported by von Economo when accounting for brain shrinkage due to fixation, and seem more accurate than other published results [4]. Future work will focus on detecting and accurately localizing FCD lesions by comparison of regional cortical thickness values of patients with the normal distributions presented in this paper.

Acknowledgements. The work was funded by grant KU Leuven IDO A99-005. We thank Alexander Hammers for making available the VOI template.

\section{References}

[1] O. Cuisenaire. Distance Transforms: Fast Algorithms and Applications to Medical Image Processing. $\mathrm{PhD}$ thesis, Laboratoire de Telecommunications et Teledetection, Université Catholique de Louvain, Louvain-la-Neuve, Belgium, 1999.

[2] B. Fischl and A.M. Dale. Measuring the thickness of the human cerebral cortex from magnetic resonance images. Proc. Natl. Acad. Sci. USA, 97(20):11050-11055, 2000.

[3] A. Hammers, M.J. Koepp, S.L. Free, M. Brett, M.P. Richardson, C. Labbé, V.J. Cunningham, D.J. Brooks, and J. Duncan. Implementation and application of a brain template for multiple volumes of interest. Human Brain Mapping, 15(3):165$174,2002$.

[4] N. Kabani, G. Le Goualher, D. MacDonald, and A.C. Evans. Measurement of cortical thickness using an autiomated 3-D algorithm: A validation study. NeuroImage, 13(2):375-380, 2001.

[5] K. Van Leemput, F. Maes, D. Vandermeulen, and P. Suetens. Automated model based tissue classification of MR images of the brain. IEEE Transactions on Medical Imaging, 18(10):897-908, 1999.

[6] D. MacDonald, N. Kabani, D. Avis, and A.C. Evans. Automated 3-d extraction of inner and outer surfaces of cerebral cortex from MRI. NeuroImage, 12(3):340-356, September 2000.

[7] F. Maes, A. Collignon, D. Vandermeulen, and P. Suetens. Multimodalatity image registration by maximization of mutual information. IEEE Transactions on Medical Imaging, 16(2):187-198, 1997.

[8] Wellcome Department of Cognitive Neurology. Statistical Parametric Mapping (SPM). http://www.fil.ion.ucl.ac.uk/spm/.

[9] R. Quester and R. Schroder. The shrinkage of the human brain stem during formalin fixation and embedding in paraffin. Journal of Neuroscience Methods., 75(1):81-89, July 1997.

[10] S.M. Sisodiya. Surgery for malformations of cortical development causing epilepsy. Brain, 123:1075-1091, 2000.

[11] C. von Economo. L'architecture cellulaire normale de l'écorce cérébrale. Masson Paris, 1927. French edition by L. Van Bogaert. 